\title{
Effect of Resource Allocation in Asymmetric Dual-hop Communication Systems
}

\author{
Woong Cho \\ Department of Computer System Engineering, Jungwon University \\ 85, Munmu-ro, Goesan-eup, Goesan-gun, Chungbuk, South Korea \\ wcho@jwu.ac.kr
}

\begin{abstract}
Relay networks have been considered as a part of next generation communication systems due to its advantages of communication range extension, capacity increment, and elimination of shadowing area. In this paper, we analyze the performance of dual-hop communication systems by considering asymmetric communication link, i.e., each sourceto-relay and relay-to-destination link has a different channel condition. The performance of the proposed system is analyzed by adopting decode-and-forward (DF) relaying protocol. The effect of resource allocation is studied to enhance the overall system performance, where the resource is categorized into relay locations and energy allocation.
\end{abstract}

Keywords: Dual-hop, relay network, asymmetric link, decode-and-forward, resource allocation,

\section{Introduction}

Relay networks can increase communication range, enhance communication capacity, and eliminate shadowing areas by transmitting signal using relay(s). Furthermore, by combining several relay links at the destination, relay networks provide cooperative diversity gain only using one antenna for each device [1-8]. Due to the benefits of relays in communication systems, they have been considered as a part of the next generation communication systems [10-15]. Basically, relay networks consist of source node, relay node(s), and destination node. The transmitted signal from the source node is received at the destination node via relay node(s). There are two representative relaying protocols in relay networks. One is the relay simply amplifies the received signal from the source node and forwards it to the destination node, and the other one is the relay demodulates the received signal from the source node and remodulates, and then forwards the remodulated signal to the destination node. The former and the later are referred as amplify-andforward (AF) and decode-and-forward (DF) relaying protocol, respectively. The performance analysis and resource allocation problems have been studied using both relaying protocols [7-9]. However, most existing work mainly considers symmetric channel scenarios, i.e., source-to-relay $(s-r)$ link and relay-to-destination $(r-d)$ links have the same channel condition. However, both channels may not always the same environment in some scenarios such as the source or destination node moves very fast. In this case one channel changes fast but the other channel may experience slow change. By motivating this situation, we consider asymmetric channel conditions in relay network. The first case is coherent-differential (C-D) scenario, where the $s-r$ link use coherent modulation scheme and $r$ - $d$ link applies differential modulation scheme. The second case is reverse of the first case, i.e., differentialcoherent (D-C) scenario, $s-r$ and $r$ - $d$ links use differential modulation scheme and

Received (April 12, 2017), Review Result (July 21, 2017), Accepted (September 15, 2017) 
coherent modulation scheme, respectively. Especially, we focus on dual-hop communication systems which use one relay node between the source node and the destination node. Figure 1 represents examples of dual-hop communications with asymmetric conditions. In Figure 1 (a), the link base station (BS)-relay-train corresponds to C-D scenario since BS-relay may have slow channel changes if the relay is static. Then, we can apply coherent modulation due to the availability of channel state information (CSI). However, the relay-train may experience fast channel change due to the fast movement of train. In this case, CSI may not be available, then we can adopt differential modulation scheme. Thus, train-relay-BS link corresponds to D-C scenario. Similar scenarios can be applied Figure 1.(b) for vehicular communication environments. By keeping mind these scenarios, we analyze the performance of dual-hop communication systems depending on relay location and energy allocation by adopting DF relaying protocol.

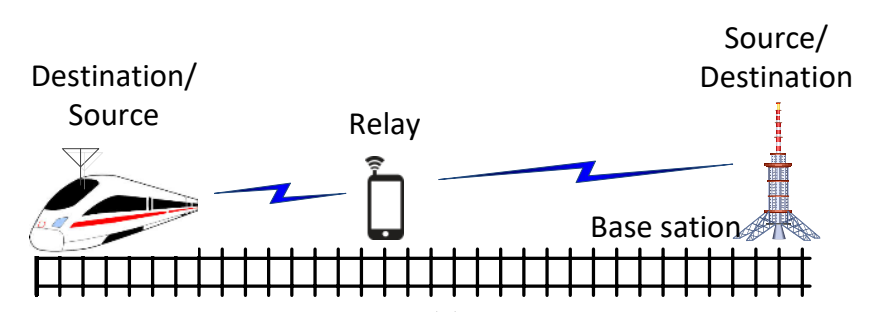

(a)

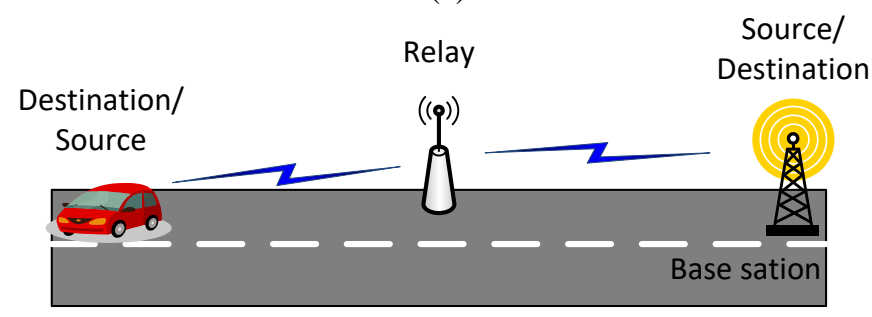

(b)

Figure 1. Application Example of Dual-Hop Communications (a) Railway Environments (B) Vehicular Environments

\section{System Model}

Figure 2 represents system model for dual-hop communication systems. Let us assume that the source node transmits signal to the destination node via the relay node. The source node transmits signal to the relay node. Since DF relaying protocol is adopted, the relay node demodulates/remodulates the received signal from the source node and forwards it to the destination node. Notice that the end node can be either a source or destination node depending on the role of the transmitter or receiver. We denote source node $s$, relay node $r$, and destination node $d$.

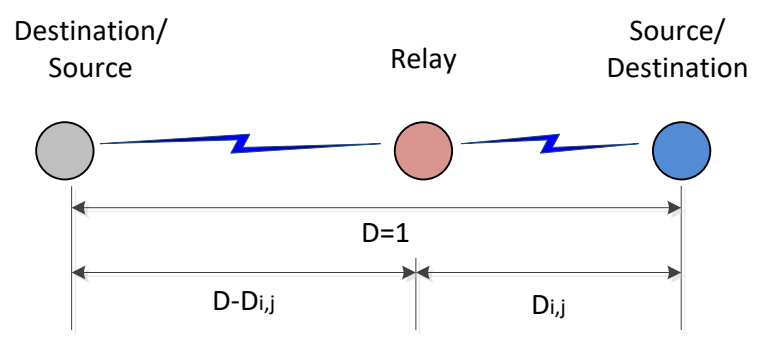

Figure 2. System Model for Dual-Hop Communications 
We let internode distance $D_{i, j}, \forall i, j \in\{s, r, d\}$ and distance between the source node and destination node $D_{s, d}=D=1$. The relay node is located between the source node and the destination node, then we have the internode relationship as $D_{s, r}+D_{r, d}=D_{s, d}$.

As we mentioned earlier, asymmetric scenarios are investigated in this paper. We consider C-D and D-C channel scenarios. The transmit signal at the source is given by

and

$$
s_{n}=e^{j 2 \pi c_{n} / M}, c_{n} \in\{0,1, \ldots, M-1\}
$$

$$
x_{n}^{s}=x_{n-1}^{s} s_{n}, x_{0}^{s}=1
$$

for the coherent modulation and differential modulation, respectively, i.e., equations (1) and (2) represent $n$th phase shift keying (PSK) symbol and the corresponding differential signal, respectively. At the relay node, the received signals for the equations (1) and (2) are given by equations (3) and (4).

$$
\begin{gathered}
y_{n, \mathrm{C}}^{s, r}=\sqrt{\varepsilon_{s}} h_{n}^{s, r} s_{n}+z_{n}^{s, r} \\
y_{n, D}^{s, r}=\sqrt{\varepsilon_{s}} h_{n}^{s, r} x_{n}+z_{n}^{s, r}
\end{gathered}
$$

The received signal is then demodulated using the corresponding modulation scheme. Let us denote demodulated symbol at the relay node as $\hat{s}_{n}^{r}$. Similar to the source transmission case, the coherent scheme transmit $\hat{s}_{n}^{r}$ directly to the destination node. Whereas, the differential scheme transmits the differentially modulated signal with $x_{n}^{r}=x_{n-1}^{r} \hat{s}_{n}^{r}, x_{0}^{r}=1$. Then the received signal at the destination node for the coherent scheme and differential scheme is given by as follows:

$$
\begin{gathered}
y_{n, \mathrm{C}}^{r, d}=\sqrt{\varepsilon_{r}} h_{n}^{r, d} \hat{s}_{n}^{r}+z_{n}^{r, d}, \\
y_{n, D}^{r, d}=\sqrt{\varepsilon_{r}} h_{n}^{r, d} x_{n}^{r}+z_{n}^{r, d} .
\end{gathered}
$$

In the above equations, $\varepsilon_{i}, i \in\{s, r\}$ represents energy per symbol at the node $i$, and fading coefficient $h_{n}^{i, j}$ and noise $z_{n}^{i, j}$ are zero-mean complex Gaussian distribution with variance $\sigma_{i, j}^{2}$ and $N_{i, j}, \forall i, j \in\{s, r, d\}$, respectively. We assume that all fading all fading coefficients are independent. We also assume that all noise components are independent and identically distributed (i.i.d) with $N_{i, j}=N_{0}, \forall i, j \in\{s, r, d\}$. Then, the instantaneous signal-to-noise ratio (SNR) and the average SNR between the transmitter $i$ and the receiver $j$ can be defined as

$$
\gamma_{i, j}=\frac{\left|h_{n}^{i, j}\right|^{2} \varepsilon_{i}}{N_{0}}
$$

and

$$
\bar{\gamma}_{i, j}=\frac{\sigma_{i, j}{ }^{2} \varepsilon_{i}}{N_{0}}
$$

for all $i, j \in\{s, r, d\}$, respectively.

At the relay node or destination node, each the received signal is demodulated depending on the corresponding modulation scheme at the receiver. Therefore, the decision rule for the coherent modulation scheme and differential modulation scheme are given by 


$$
\hat{S}_{n}=e^{j 2 \pi m_{n}^{\prime} / M}: m_{n}^{\prime}=\underset{m \in 0,1, \ldots, M-1}{\arg \max } \mathfrak{R}\left\{\left(h_{n}^{i, j}\right)^{*}\left(y_{n, C}^{i, j}\right) e^{j 2 \pi m / M}\right\}
$$

and

$$
\hat{S}_{n}=e^{j 2 \pi m_{n}^{\prime} / M}: m_{n}^{\prime}=\underset{m \in 0,1, \ldots, M-1}{\arg \max } \mathfrak{R}\left\{\left(y_{n-1, D}^{i, j}\right)^{*}\left(y_{n, D}^{i, j}\right) e^{j 2 \pi m / M}\right\}
$$

for all $i, j \in\{s, r, d\}$, respectively. In equations (9) and (10), $\mathfrak{R}(\cdot)$ and $(\cdot)^{*}$ represent real part and complex conjugate of signal, respectively.

\section{Simulations}

As we noted in Section 1, we consider dual-hop environments. To capture the effect of relay location, we use the relationship between the average power of channel fading coefficient and the internode distance $D_{i, j}$ as follows:

$$
\sigma_{i, j}^{2}=C \cdot D_{i, j}^{-\alpha}, i, j \in\{s, r, d\}
$$

where $\alpha$ is the path loss exponent of wireless channel, and $\mathrm{C}$ is a constant which we set to 1 without loss of generality. In simulations, we set $\alpha=4$ for all cases.To analyze the effect of energy allocation, we constraint the total energy as follows:

$$
\varepsilon_{s}+\varepsilon_{r}=\varepsilon \text {. }
$$

By defining the total SNR as $\rho=\varepsilon / N_{0}$, the transmit SNR $\rho_{s}=\varepsilon_{s} / N_{0}$ and the transmit SNR at the relay node $\rho_{r}=\varepsilon_{r} / N_{0}$, the total energy constraint can be written as follows:

$$
\rho=\rho_{s}+\rho_{r} .
$$

Using the equations (8) and (11), the average SNR can be expressed in terms of the transmit SNR's as:

$$
\bar{\gamma}_{s, r}=\rho_{s} \sigma_{s, r}^{2}=\rho_{s} D_{s, r}^{-\alpha}
$$

and

$$
\bar{\gamma}_{r, d}=\rho_{r} \sigma_{r, d}^{2}=\rho_{r} D_{r, d}^{-\alpha} .
$$

Then the total energy constraint can be re-expressed as

$$
\rho=\bar{\gamma}_{s, r} / \sigma_{s, r}^{2}+\bar{\gamma}_{r, d} / \sigma_{r, d}^{2}=\bar{\gamma}_{s, r} D_{s, r}^{\alpha}+\bar{\gamma}_{r, d} D_{r, d}^{\alpha} .
$$

Based on the above total energy constraint and internode distant condition, the effect of relay location and energy allocation is analyzed. Figure 3 depicts the effect of relay location where we move the relay node from the source node to the destination node by considering various total energy constraint. The $x$ axis and $y$ axis represent the location of relay node and SER, respectively. The figure shows that the SER trend of energy allocation is the same regardless of the total SNR. However, the optimum location of relay node which provides minimum SER is different for the channel scenario. The minimum SER can be achieved by selecting the relay node approximately 0.55 and 0.45 for C-D scenario and D-C scenario, respectively. These results are different from the symmetric scenarios, i.e., C-C and D-D cases, in [7-9]. 


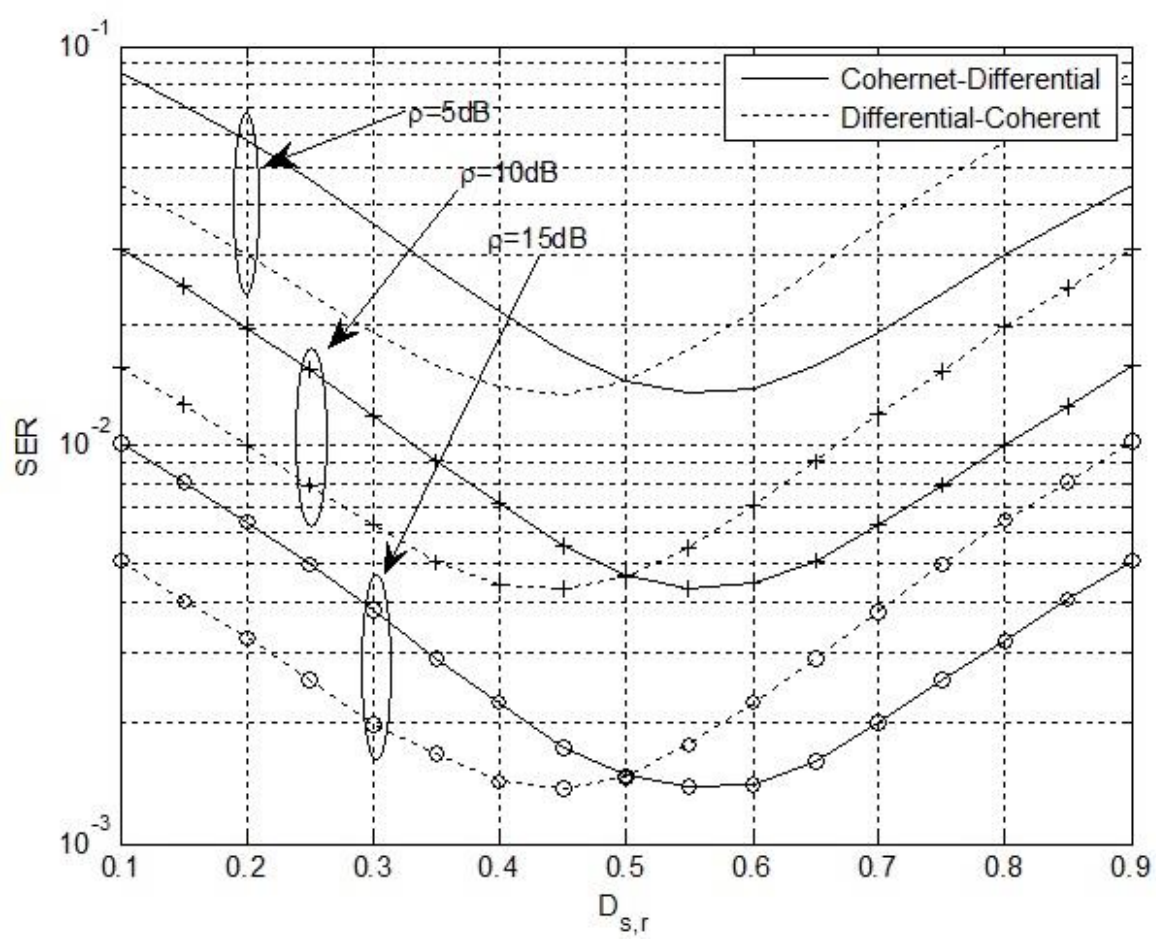

Figure 3. SER Depending On Relay Location

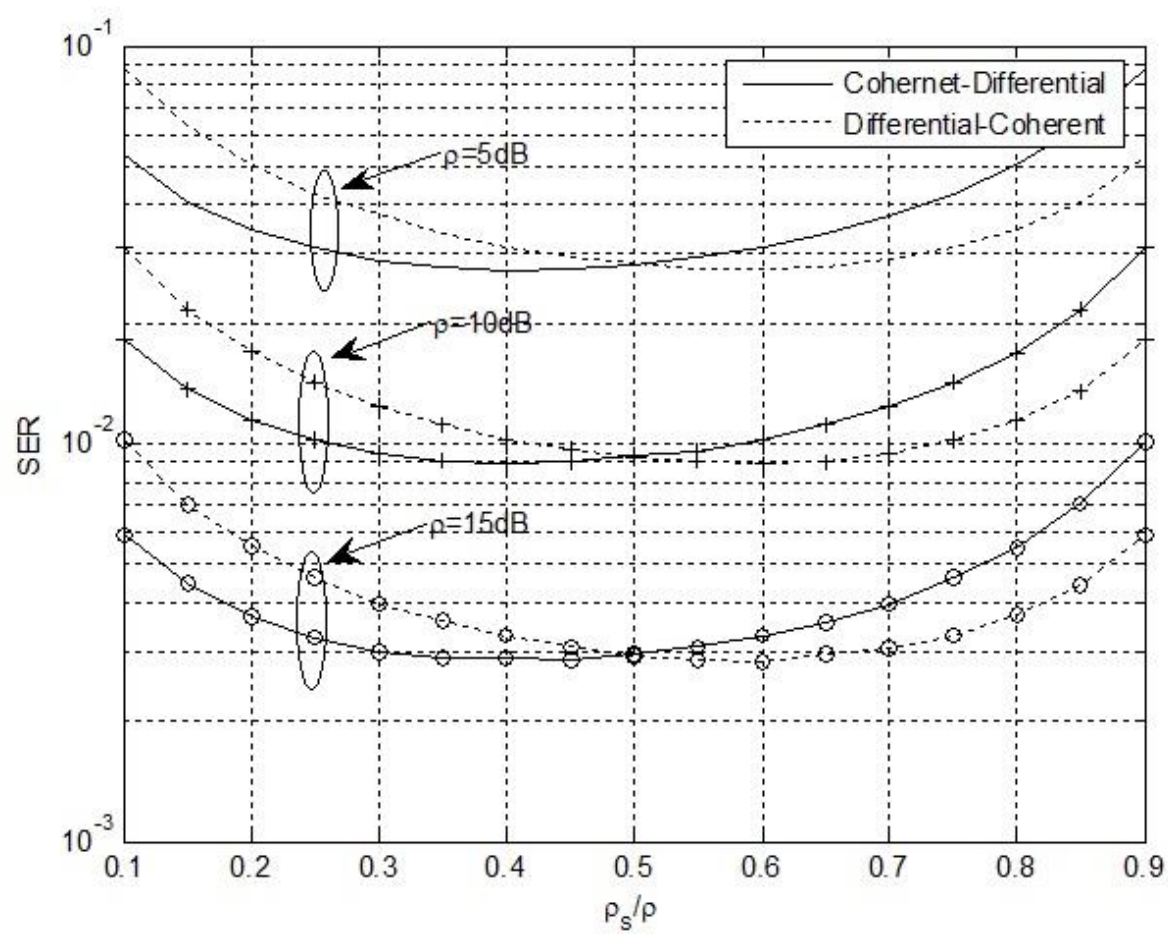

Figure 4. SER Depending On Energy Allocation

Figure 4 represents the effect of energy allocation where we assign $10 \%$ of total energy to the source node and change the percentage up to $90 \%$. Similar to the location allocation case, the strength of SNR does not affect the trend of performance. The figure shows that minimum SER can be provided when approximately $40 \%$ and $60 \%$ of total energy are assigned at the source node for C-D 
scenario and D-C scenario, respectively. From the two figures, we can see that energy allocation has more flat SER variation than location allocation, i.e., the location allocation is more sensitive than the energy allocation.

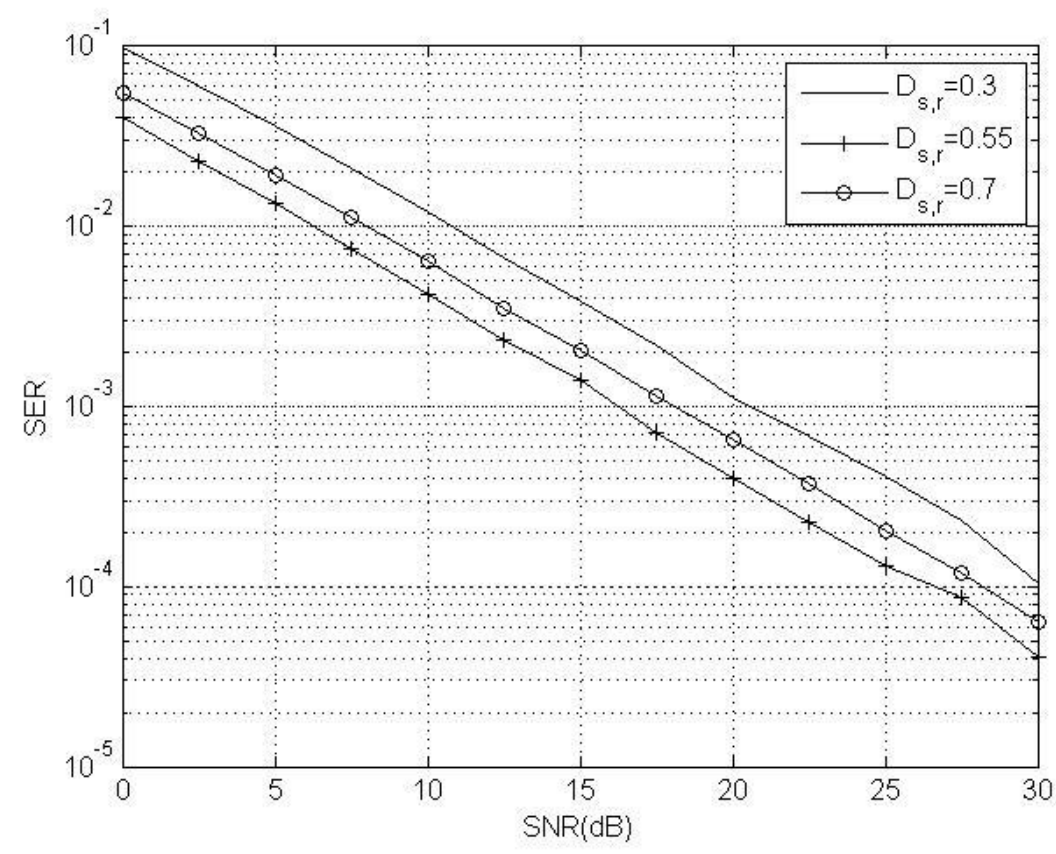

Figure 5. SER for the Given Relay Location: C-D Scenario

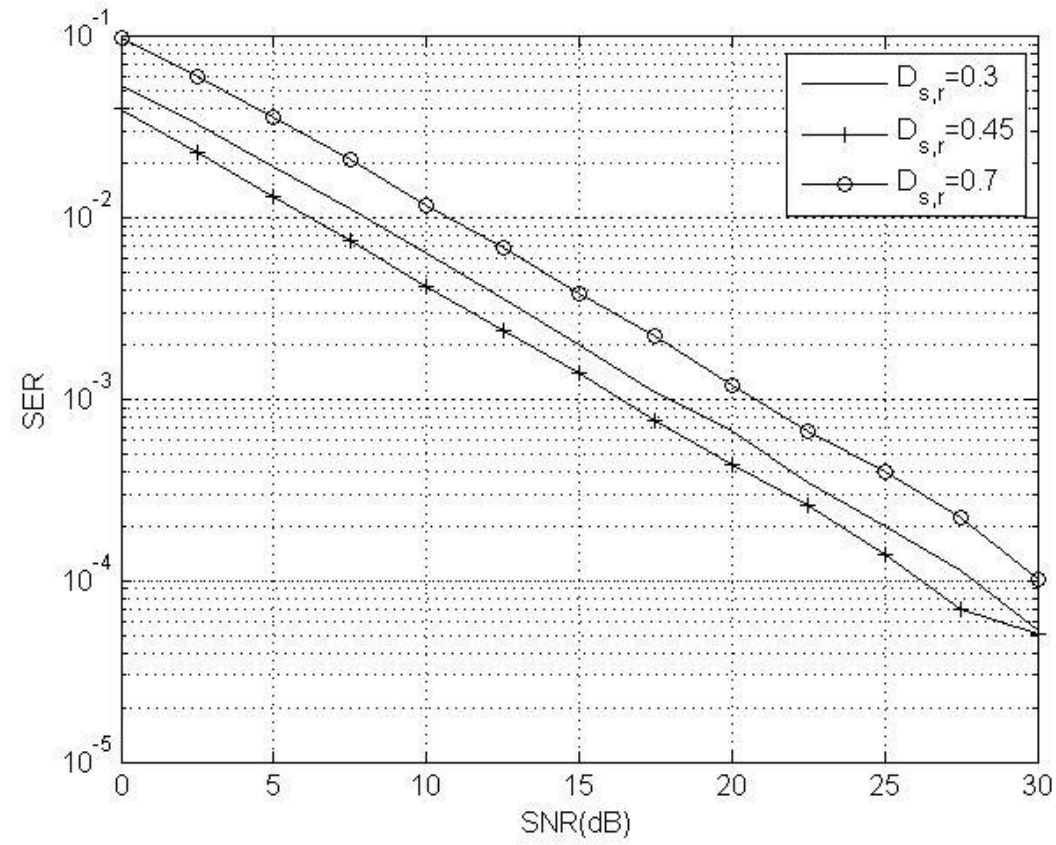

Figure 6. SER for the Given Relay Location: D-C Scenario

Figures 5 and 6 represent SER of dual-hop communication systems for the given relay locations. The relay locations are selected three cases: the optimum point in Figure 3, the near source location, and the near destination location. Then, SER with the various SNRs is demonstrated for both C-D and D-C scenarios. The figures 
show that the minimum SER is well matched with the result in Figure 3, which implies that we can achieve better performance by locating or selecting the relay node at the specific point.

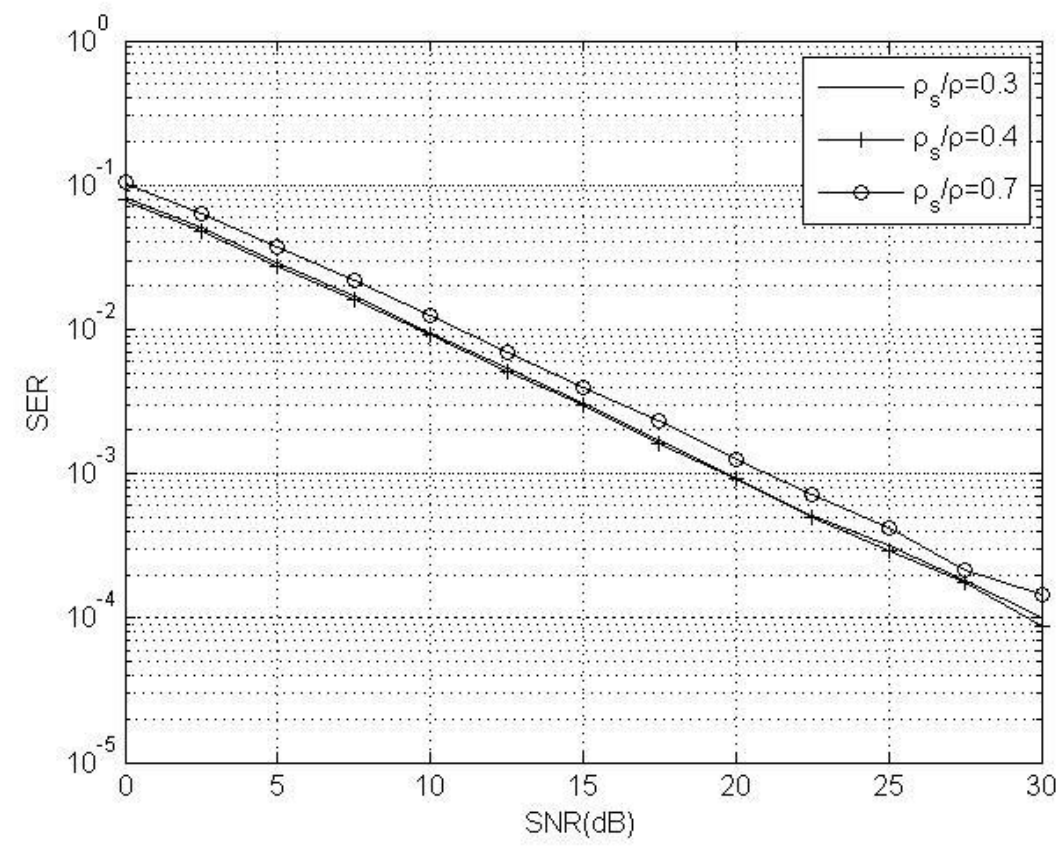

Figure 7. SER for the Given Energy Allocation: C-D Scenario

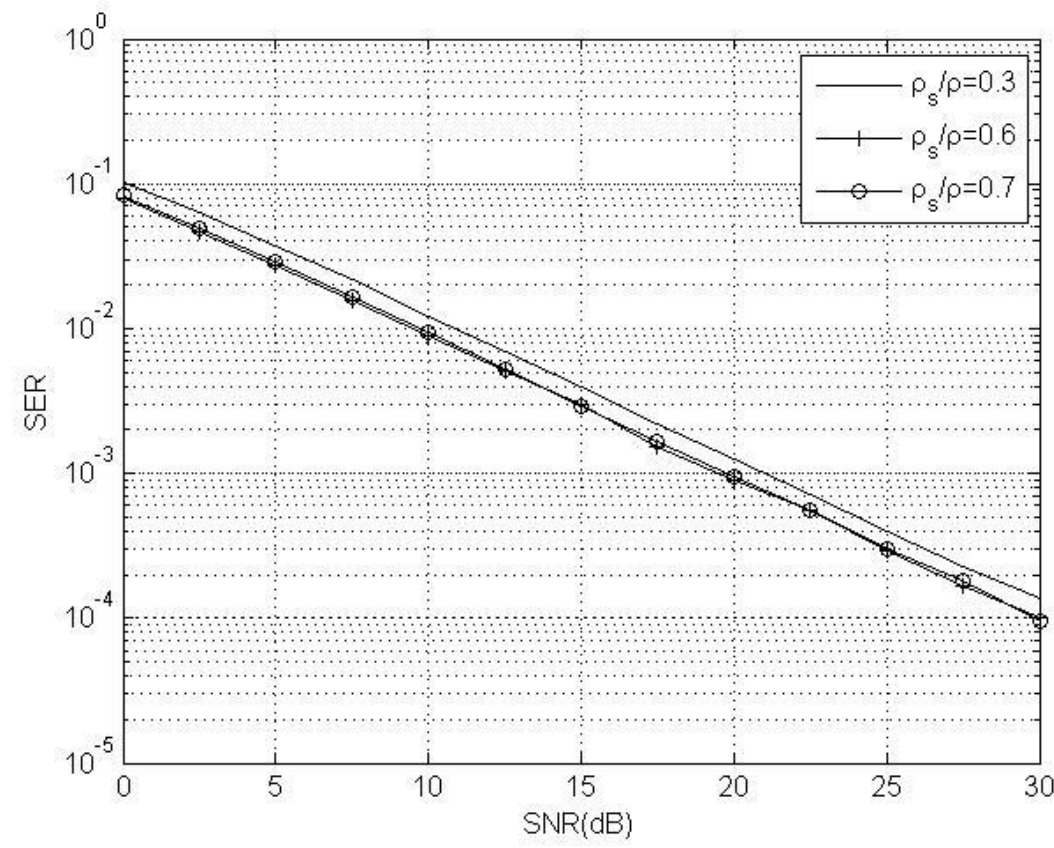

Figure 8. SER for the Given Energy Allocation: D-C Scenario

Figures 7 and 8 represent SER of dual-hop communication systems for the given energy allocation by assuming the total transmit energy is limited. Similar to the location allocation case, we select three transmit energy cases: the optimum point in 
Figure 4 , more energy is allocated at the destination $\operatorname{node}\left(\rho_{s} / \rho=0.3\right)$, and more energy is allocated at the source node $\left(\rho_{s} / \rho=0.7\right)$. Then, SER with the various SNRs is considered for both C-D and D-C scenarios. The results show that the minimum SER is can be achieved by selecting the energy allocation in Figure 4. However, the performance differences for three cases are much smaller than location allocation case. This implies the location allocation is more critical than the energy allocation, i.e., location information is more sensitive to control the performance of dual-hop communication systems. This result is well agreed with the results in Figures 3 and 4. Therefore, in practical implementations, we may enhance the performance by choosing a proper relay node based on the results in this paper when several relay nodes exist.

\section{Conclusions}

In this paper, we explore the effect of energy and location allocation for asymmetric dual-hop communication systems. The results reveal that the optimum point which shows minimum SER is different for the C-D scenario and D-C scenario. For the relay location case, we can achieve better performance by choosing the relay node little bit shift to the destination node from the midpoint for the C-D scenario, and vice versa for the $\mathrm{D}-\mathrm{C}$ scenario. For the energy allocation case, the minimum SER can be achieved by assigning little more energy for the relay node for C-D scenario, and vice versa for the D-C scenario. These results are different from the symmetric cases. We also reveal that the effect of energy allocation in the overall performance is less than the location allocation.

\section{Acknowledgments}

This work was supported by the Jungwon University Research Grant 2016-33.

\section{References}

[1] J. N. Laneman and G. W. Wornell, "Energy-efficient Antenna Sharing and Relaying for Wireless Networks", Proceeding of Wireless Communication and Networking Conference, Chicago, IL, (2000).

[2] J. N. Laneman, D. N. C. Tse and G. W. Wornell, "Cooperative Diversity in Wireless Networks: Efficient Protocols and Outage Behavior", IEEE Transactions on Information Theory, vol. 50, no. 12, (2004), pp. 3062-3080.

[3] A. Ribeiro, X. Cai and G. B. Giannakis, "Symbol Error Probabilities for General Cooperative Links", IEEE Transactions on Wireless Communications, vol. 4, no. 3, (2005), pp. 1264-1273.

[4] D. Chen and J. N. Laneman, "Modulation and Demodulation for Cooperative Diversity in Wireless Systems", IEEE Transactions on Wireless Communications, vol. 5, no. 7, (2006), pp. 1785-1794

[5] Y. Lee, M-H. Tsai and S-I Sou, "Performance of Decode-and-Forward Cooperative Communications with Multiple Dual-Hop Relays over Nakagami-m Fading Channels", IEEE Transactions on Wireless Communications, vol. 8, no. 6, (2009), pp. 2853-2859.

[6] S. S. Ikki and M. H. Ahmed, "Performance Analysis of Adaptive Decode-and-Forward Cooperative Diversity Networks with Best-Relay Selection”, IEEE Transactions on Communications, vol. 58, no. 1, (2010), pp. 68-72.

[7] W. Cho and L. Yang, "Optimum resource allocation for relay networks with differential modulation", IEEE Transactions on Communications, vol. 56, no. 4, (2008), pp. 531-534.

[8] W. Cho, R. Cao and L. Yang, "Optimum resource allocation for amplify-and-forward relay networks with differential modulation", IEEE Transaction on Signal Processing, vol. 56, no. 11, (2008), pp.56805691.

[9] R. Cao and L. Yang "The affecting factors in resource optimization for cooperative communications: A case study", IEEE Transaction on Wireless communications, vol. 11, no. 12, (2012), pp.4351-4361.

[10] J. Calle-Sanchez, M. Molina-Garcia, J. I. Alonso and A. Fernandez-Duran, "Long Term Evolution in high speed railway environments: feasibility and challenges", Bell Labs Technical Journal, vol. 18, no. 2, (2013), pp.237-253.

[11] 3GPP TR 22.803: Feasibility study for proximity services (ProSe) (2013). 
[12] M. Iwamura, H. Takahashi and S. Nagata, "Relay technology in LTE-advanced", NTT Docomo Technical Journal, vol. 18, no. 2, (2010), pp. 31-36.

[13] M. N. Tehrani, M. Uysal and H. Yanikomeroglu, "5G cellular networks: challenges, solutions, and future directions", IEEE Communication Magazine, vol. 52, no. 5, (2014), pp. 86-92.

[14] S. Lien, C. Chien, G. S. Liu, H. Tsai, R. Li and Y. J. Wang, "Enhanced LTE device-to-device proximity services", IEEE Communication Magazine, vol. 54, no.12, (2016), pp. 174-182.

[15] F. H. Kumbhar, N. Saxena and A. Roy, "Reliable relay; autonomous social DeD paradigm for 5G LoS communications", IEEE Communications letters, vol. 21, no. 7, (2017), pp. 1593-1596.

\section{Author}

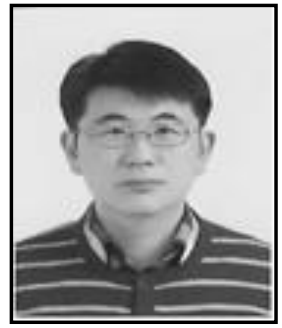

Woong Cho, Woong Cho received the B.S. degree in electronics engineering from the University of Ulsan, Ulsan, South Korea, in 1997, the M.S. degrees in electronic communications engineering and electrical engineering from Hanyang University, Seoul, South Korea, in 1999, and the University of Southern California, Los Angeles, in 2003, respectively, and the Ph.D. degree in electrical and computer engineering from University of Florida, Gainesville, in 2007. He was a senior research engineer from Feb. 2008 to Feb. 2012 in Electronics and Telecommunications Research Institute (ETRI), Daejeon, South Korea. He is currently with the department of Computer System Engineering in Jungwon University, Goesan, South Korea. His research interests are cooperative/relay networks, vehicular communications, and signal processing for wireless communications. 
International Journal of Control and Automation

Vol. 10, No. 11 (2017) 